FIAN/TD-03/2000

BNL-NT-00/13

\title{
AZIMUTHAL ASYMMETRY IN TRANSVERSE ENERGY FLOW IN NUCLEAR COLLISIONS AT HIGH ENERGIES
}

\author{
Andrei Leonidov $^{(a),(b)}$ and Dmitry Ostrovsky ${ }^{(b)}$ \\ (a) Physics Department, Brookhaven National Laboratory \\ Upton, NY 11973, USA \\ (b) Theoretical Physics Department, P.N. Lebedev Physics Institute \\ 117924 Leninsky pr. 53, Moscow, Russia円
}

\begin{abstract}
The azimuthal pattern of transverse energy flow in nuclear collisions at RHIC and LHC energies is considered. We show that the probability distribution of the event-by-event azimuthal disbalance in transverse energy flow is essentially sensitive to the presence of the semihard minijet component.
\end{abstract}

\footnotetext{
${ }^{1}$ Permanent address
} 
At the current level of understanding of the strong interaction physics, any attempt to give a quantitative description of the bulk of the events in high energy hadron and nuclear collisions necessarily invokes several dynamical sources of inelasticity (transverse energy production), see e.g. [1]. When materialized in the form of a Monte-Carlo event generator such as PYTHIA [2] or HIJING [3], the description of primordial production of partonic degrees of freedom, quarks and gluons, and of their subsequent conversion into observable hadrons is necessarily model-dependent. This refers in particular to specific assumptions made about the contribution of multiple parton collisions, description of the final state and especially initial state radiation, etc. At the same time the presence of new physics brought in by semihard degrees of freedom should manifest itself through reasonably well-defined changes in the inelasticity pattern that can (hopefully) be measured experimentally. Ideally the set of such specific measurements should allow to discriminate between various otherwise successful approaches (e.g. minijet physics vs. dual parton model). Extensive discussion of the relevance of semihard degrees of freedom (minijets) in explaining such features of high energy hadron collisions as the enhanced tails of the multiplicity distributions, multiplicity dependence of the mean transverse energy, etc. by exploiting a realistic Monte-Carlo event generator HIJING can be found in [3] and in the review 4 .

One particular aspect, discussed in [5], is a $p_{\perp}$ dependence of the two-particle correlation function due to the semihard minijet contribution in $p \bar{p}$ collisions. The detailed analysis of the minijet contribution to the two-particle correlation function in heavy ion collisions will appear in the forthcoming publication [6]. Below a related calorimetric measure of the specific event-by-event azimuthal correlations in the transverse energy flow due to minijet contribution will be discussed. The consideration closely follows the analysis performed in [7], where a specific model of minijet dynamics based on leading twist collinearly factorized QCD was considered. The main goal of the present analysis is, by using the HIJING event generator, include the mechanisms beyond the simple picture of binary parton-parton collisions considered in [7] such as multiple parton collisions, initial and final state radiation accompanying these collisions, soft contributions to transverse energy due to hadronization, jet quenching in nuclear collisions, etc. Let us note, that accounting for these mechanisms is crucial for reproducing the transverse energy spectrum [8]

In the context of ultrarelativistic heavy ion collisions the primordial system of semihard degrees of freedom (minijets) is setting a stage for subsequent (possibly collective) evolution [9]. Different approaches are being used to study the properties of the initial conditions in high energy nuclear collisions. The conceptually simplest one is based on accounting for lowest order perturbative contributions in collinearly factorized QCD, see e.g. the recent papers [10] and references therein. Another approach, based on the quasiclassical treatment of the gluon fields in the colliding nuclei, was considered in a number of publications [1]. A program of the non-perturbative analysis of primordial gluon distributions is being realized in refs. [12]. Finally, one can rely on the description of minijet effects as implemented in the realistic Monte-Carlo generators such as PYTHIA and HIJING, where effects of all orders in perturbation theory are (effectively) taken into 
account. In particular, calculations with HIJING allow to study the effects due to the presence of semihard degrees of freedom at the early stages of high energy collision in a simple setting, where the only nontrivial effect distinguishing the nuclear collision from an incoherent superposition of nucleon-nucleon ones is jet quenching, i.e. energy losses experienced by partons traversing the surrounding debris created in nuclear collision.

To quantify the event-by-event asymmetry of transverse energy flow, we consider, following [7], the difference between the transverse energy deposited, in some rapidity window $y_{\min }<y_{i}<y_{\max }$, into two oppositely azimuthally oriented sectors with a specified angular opening $\delta \varphi$ each 2 .

For convenience one can think of the directions of these cones as being "up" and "down" corresponding to some specific choice of the orientation of the system of coordinates in the transverse plane. All results are, of course, insensitive to the particular choice. Denoting now the transverse energy going into the "upper" and "lower" cones in a given event by $E_{\perp}^{\uparrow}(\delta \varphi)$ and $E_{\perp}^{\downarrow}(\delta \varphi)$ correspondingly, we can quantify the magnitude of the asymmetry in transverse energy production in a given event by

$$
\delta E_{\perp}(\delta \varphi)=E_{\perp}^{\uparrow}(\delta \varphi)-E_{\perp}^{\downarrow}(\delta \varphi)
$$

its statistical properties characterized by the corresponding probability distribution

$$
P\left(\delta E_{\perp} \mid \delta \varphi\right)=\frac{d w\left(\delta E_{\perp}(\delta \varphi)\right)}{d \delta E_{\perp}(\delta \varphi)}
$$

We have calculated this distribution in HIJING for central AuAu collisions at RHIC energy $\sqrt{s}=200 \mathrm{GeV}$ and central $\mathrm{PbPb}$ collisions at LHC energy $\sqrt{s}=5.5 \mathrm{TeV}$ for $\delta \varphi=\pi$. The distributions $P\left(\delta E_{\perp} \mid \pi\right)$ have been calculated both at partonic level and at the level of final hadrons with semihard interactions and quenching on and off. This allowed us to study the contribution of HIJING minijets and of the effects of their hadronization to the asymmetry in question. The resulting distributions are plotted in Figs. 1 and 2, for RHIC and LHC energies respectively with quenching turned on and the value of the minijet's infrared cutoff $p_{0}=2 \mathrm{GeV}$.

The numerical values of the mean square deviation $\delta E_{\perp}$ characterizing the widths of the corresponding probability distributions in Figs. 1 and 2 are given in Table 1, where for completeness we also give the widths for the probability distributions with quenching turned off and with a larger value for the infrared cutoff $p_{0}=4 \mathrm{GeV}$

\footnotetext{
${ }^{2}$ Long-range correlations in the polar (rapidity) energy flow in heavy ion collisions were recently considered in 13 .
} 


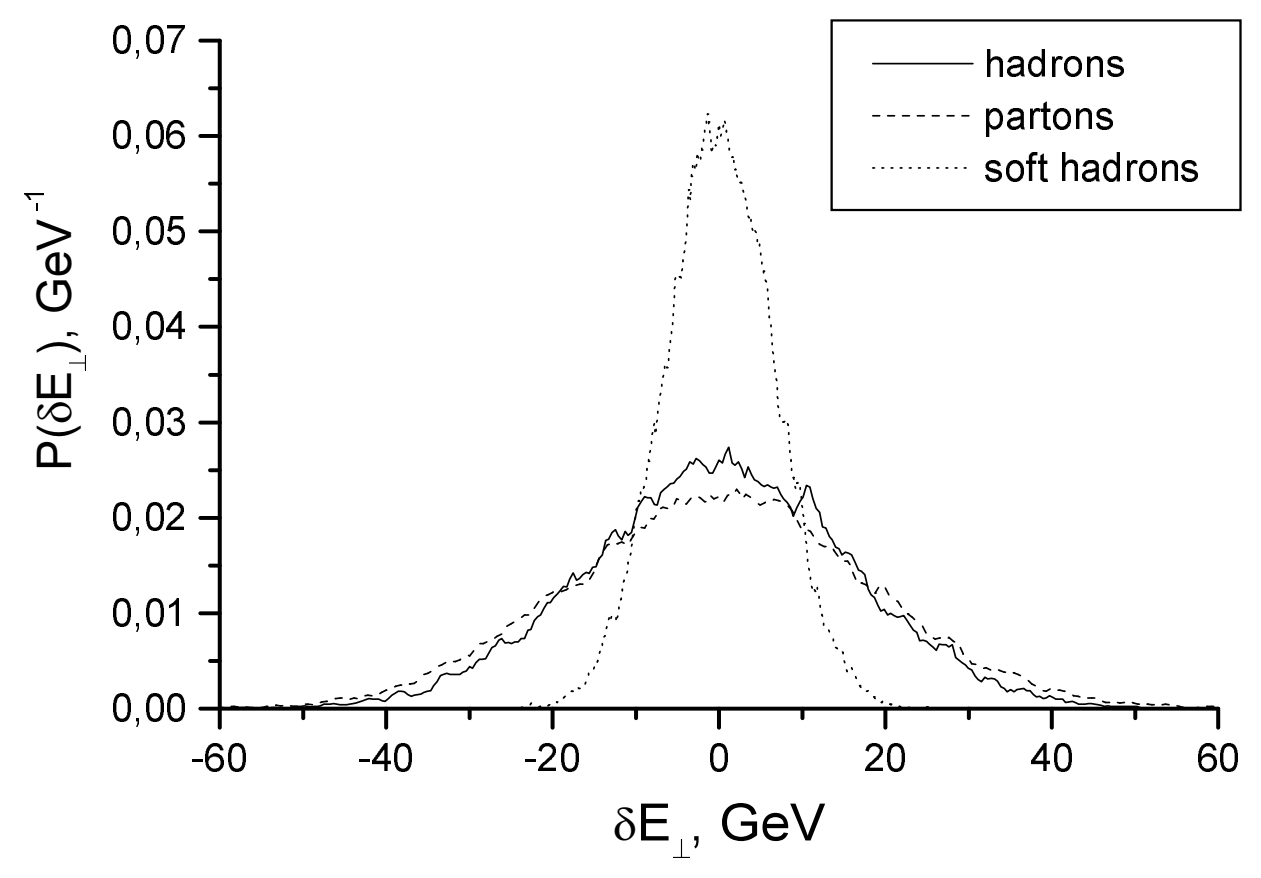

Figure 1: Probability distribution for azimuthal transverse energy disbalance in the unit rapidity window for $\mathrm{AuAu}$ collisions at RHIC energy $\sqrt{s}=200 \mathrm{GeV}, p_{0}=2 \mathrm{GeV}$, quenching on.

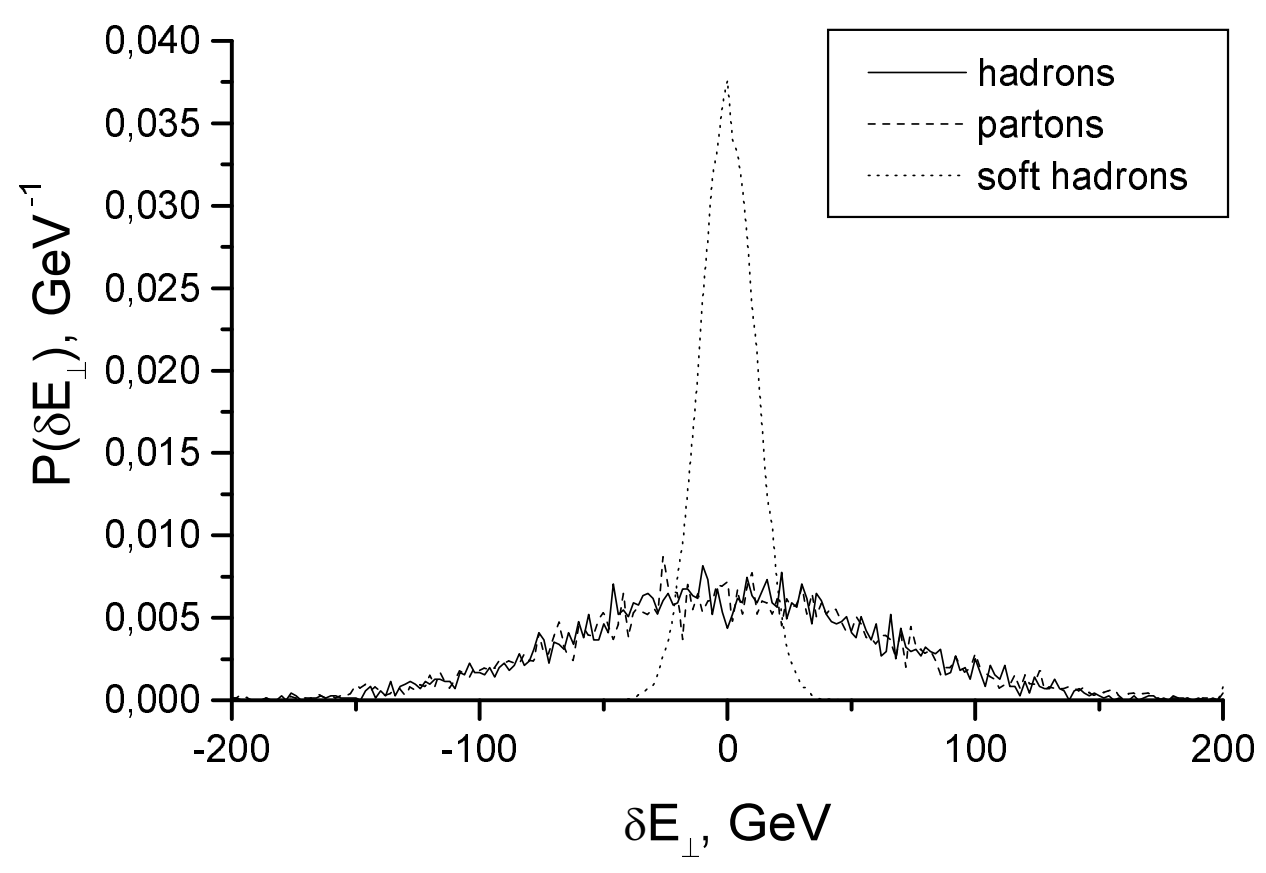

Figure 2: Probability distribution for azimuthal transverse energy disbalance in the unit rapidity window for $\mathrm{PbPb}$ collisions at LHC energy $\sqrt{s}=5.5 \mathrm{TeV}, p_{0}=2 \mathrm{GeV}$, quenching on. 


\begin{tabular}{|c|c|c|c|c|}
\hline $\mathrm{AA}$ & $\sqrt{S}, \mathrm{GeV}$ & $p_{0}, \mathrm{GeV}$ & asymmetry & $\sqrt{\left\langle\delta E^{2}\right\rangle}$ \\
\hline \multirow{4}{*}{$\mathrm{AuAu}$} & \multirow{4}{*}{200} & \multirow{4}{*}{2} & hadrons (quenching on) & 16 \\
\hline & & & hadrons (quenching off) & 17 \\
\hline & & & partons & 18 \\
\hline & & & soft hadrons & 7 \\
\hline \multirow{4}{*}{$\mathrm{PbPb}$} & \multirow{4}{*}{5500} & \multirow{4}{*}{2} & hadrons (quenching on) & 61 \\
\hline & & & hadrons (quenching off) & 71 \\
\hline & & & partons & 65 \\
\hline & & & soft hadrons & 15 \\
\hline \multirow{3}{*}{$\mathrm{PbPb}$} & \multirow{3}{*}{5500} & \multirow{3}{*}{4} & hadrons (quenching on) & 69 \\
\hline & & & partons & 76 \\
\hline & & & soft hadrons & 16 \\
\hline
\end{tabular}

Table 1

The main conclusions that can be drawn from Figs. 11 and 2 and Table 1 are the following.

First, the magnitude of the azimuthal asymmetry as measured by the width of the probability distribution $P\left(\delta E_{\perp} \mid \delta \varphi\right)=d w\left(\delta E_{\perp}(\delta \varphi)\right) / d \delta E_{\perp}(\delta \varphi)$ is essentially sensitive to semihard interactions (minijets). Switching off minijets, and thus restricting oneself to purely soft mechanisms, leads to a substantial narrowing of the asymmetry distribution; by the factor of 2.3 at RHIC and by the factor 4.1 at LHC energy rspectively (these values correspond to the case of quenching being turned on).

Second, quite remarcably, the parton and final (hadronic) distributions of $\delta E_{\perp}$ in both cases practically coincide indicating that the contribution to transverse energy due to hadronization of the initial parton system is, with a high accuracy, additive and symmetric in between the oppositely oriented cones. Both conclusions show that the energy-energy correlation in Eq. (1) is a sensitive measure of the primordial parton dynamics that can be studied in calorimetric measurements in central detectors at RHIC and LHC.

Third, as expected, turning off quenching somewhat enhances the fluctuations. However, as seen from the table, numerically the effect is not important. This shows once again that the proposed asymmetry is really essentially determined by the earliest stage of the collision, when the primordial parton flux is formed.

Finally, from Table 1 we conclude that the studied asymmetry is not particularly sensitive to changing the value of the infrared cutoff $p_{0}$ and thus provides a robust signal of the presence of semihard dynamics.

\section{Acnowledgements}

A.L. thanks J.-Y. Ollitrault for useful discussions.

A.L. is grateful to Laboratoire de Physique Theoriquie, Saclay, where this work was started, and BNL nuclear theory group, where it was completed, for kind hospitality. This manuscript has been authored under Contract No. DE-AC02-98CH10886 with the U.S. Department of Energy.

The work was partially supported by RFBR grant 00-02-16101 


\section{References}

[1] T. Sjostrand and M. van der Zijl, Phys. Rev. D36 (1987), 2019

[2] T. Sjostrand, Comput. Phys. Commun. 82 (1994), 74;

[3] X.-N. Wang and M. Guylassy, Phys. Rev. D44 (1991), 3501; D45 (1992), 844; Comput. Phys. Commun. 83 (1994), 307

[4] X.-N. Wang, Phys. Repts 280 (1997), 287

[5] X.-N. Wang, Phys. Rev. D46 (1992), R1900; D47 (1993), 2754

[6] A. Leonidov and J.-Y. Ollitrault "On Azimuthal Correlations in Heavy Ion Collisions", in preparation

[7] A. Leonidov and D. Ostrovsky, "Angular Pattern of Minijet Transverse Energy Flow in Hadron and Nuclear Collisions", hep-ph/9812416

[8] A. Leonidov, "On Transverse Energy Production in Hadron Collisions", BNL-NT$00 / 12$

[9] J.P. Blaizot and A.H. Mueller, Nucl. Phys. B289 (1987), 847

[10] K.J. Eskola, K. Kajantie, P.V. Ruuskanen and K. Tuominen, Nucl. Phys. B570 (2000), 379;

K.J. Eskola, K. Kajantie and P.V. Ruuskanen, Eur. Phys. J. C1 (1998), 627;

[11] A. Kovner, L. McLerran and H. Weigert, Phys. Rev. D52 (1995), 3809;

Phys. Rev. D52 (1995), 6231;

Yu. V.Kovchegov and D.H. Rischke, Phys. Rev. C56 (1997), 1084

S.G. Matinyan, B. Mueller and D.H. Rischke, Phys. Rev. C56 (1997), 1927;

Phys. Rev. C57 (1998), 2197;

M. Gyulassy and L. McLerran, Phys. Rev. C56 (1997), 2219

[12] A. Krasnitz and R. Venugopalan, Nucl. Phys. B557 (1999), 237;

"Real time simulations of high energy nuclear collisions", [hep-ph/9808322];

"Making Glue in High-Energy Nuclear Collisions", hep-ph/9905319;

"The Initial Energy Density of Gluons Produced in Very High Energy Nuclear Collisions", [hep-ph/9909203];

"The First Fermi in a High Energy Nuclear Collision", hep-ph/9910391;

"Nonperturbative Gluodynamics of High Energy Heavy-Ion Collisions", hep-ph/0004116

[13] Yu.V. Kovchegov, E. Levin and L. McLerran, "Large Scale Rapidity Correlations in Heavy Ion Collisions", hep-ph/9912367 